\title{
Young's Modulus and Loss Factor Estimation of Sandwich Beam with Three Optimization Methods by FEM
}

\author{
Key Fonseca de \\ Lima \\ Dept. Mech. \\ Engineering \\ PPGEM \\ PUCPR \\ Curitiba,Paraná
}

Brazil

\author{
Nilson Barbieri \\ Dept. Mech. \\ Engineering \\ PPGEM \\ PUCPR \\ Curitiba,Paraná \\ Brazil
}

\author{
Renato Barbieri \\ Dept. Mech. \\ Engineering \\ UDESC \\ Joinville Santa \\ Catarina \\ Brazil
}

\author{
Luiz C. Winniques \\ Dept. Mech. \\ Engineering \\ PPGEM \\ PUCPR \\ Curitiba,Paraná \\ Brazil
}

\begin{abstract}
This work aims to propose an inverse methodology for the physical properties identification of sandwich beams by measured flexural resonance frequencies. The physical parameters are the Young's modulus and the loss factor. They are estimated for each one of materials that constitute the structure of the sandwich beam which is made with the association of Hot-rolled steel, Polyurethane Rigid Foam and High Impact Polystyrene. This kind of the sandwich beam are widely used for the assembly of household refrigerators and food freezers. The solutions are obtained with parametric optimization of physical parameters of the materials that forming the sandwich beam with three methods: Genetic Algorithms (GA), Differential Evolution (DE), and Particle Swarm Optimization (PSO). Furthermore, this work intend verify the quality of the solutions obtained with parametric optimization. The parameters are estimated using measured and numeric frequency response functions (FRFs). The mathematical model to verify numeric FRF is obtained using the Finite Element Method and the two-dimensional elasticity theory coupled to three optimization methods. The results of the optimizations show that it is possible to determine effectively the physical parameters of a sandwich beam with this methodology.
\end{abstract}

\section{Keywords}

Sandwich beam, Optimization, Young's Modulus, GA, PSO, DE.

\section{INTRODUCTION}

This work aims to propose an inverse methodology for the physical properties identification of sandwich beams by measured flexural resonance frequencies. The solutions are obtained with parametric optimization of physical parameters of the materials that forming a sandwich beam with three methods: Genetic Algorithms (GA), Differential Evolution (DE), and Particle Swarm Optimization (PSO). In this case it was used mathematical model obtained by finite element method (FEM) with two dimensional (2D) elasticity theory and a objective function based on the difference between experimental and numerical values of ressonance frequencies. The parameters estimated were the Young's modulus and the loss factor of the Polyurethane Rigid Foam (PRF) and High Impact Polystyrene (HIP). The physical parameters of sandwich beams made with the association of hot-rolled steel, PRF and HIP applied to assembly of household refrigerators and food freezer are estimated using measured and numeric frequency response functions (FRFs).
As already exposed, the mathematical model is obtained by FEM with the 2D elasticity theory with the following characteristics:

a)The sandwich beam is discretized with cubic elements which are suitable to represent the shear stress along the thickness of beam. This does not provided with lower order elements. The test sample is a beam that can be considered short and the amount of strain energy associated with the shear is important in the calculations;

b)As the core of sandwich beam is softer than the face, appear regions of compression/traction core vibration modes that can not be represented by the classical theory of Timoshenko or Euler-Bernoulli beam. This compression/traction is best observed in higher order modes;

c) $2 \mathrm{D}$ element can prescribe regions of detachment and 2D model provides better representation of the boundary conditions (clamped end);

d)This model is closer to reality which leads to adjustments that are more accurate;

e)Disadvantage, the 2D model needs high computational time (mesh with more degrees of freedom). How $\omega$ (angular frequency) is variable, for each new value of this parameter a new finite element analysis should be performed, because the equivalent stiffness matrix depends on $\omega$.

The GA is based on Darwin's concept of the selection and natural evolution applied to mathematical programming. It was originally proposed by Holland and Jong in 1975 [1] and it has been widely used in the last years for obtaining optimum solutions for many engineering efforts. The GA has the ability of searching for a global optimum in discontinuous and multimodal spaces without requiring a proper initial point [2]. The DE is a direct search method that was developed for minimizing the problems represented by non-linear and non-differentiable continuous spatial functions. This method was created in 1995 by Storn and Price [3] when attempting to solve a polynomial fitting problem by Chebychev associated with electronic filter designs. In addition, the method is fairly simple and presents fast convergence features for problems that involve a small number of particles and a large number of design variables. The PSO was introduced by Eberhart and Kennedy in 1995 [4], using concepts of social behavior of populations, such as bird flocking and fish schooling. The PSO is an optimization technique based on just one population with predetermined number of the particles that evolve within the hyperspace 
defined by the design variable boundaries following some random criteria towards the particle with the best performance (usually the particle that is closest to the optimum point). The application these three methods for physical properties identification of materials can be seen in Quaranta et al. [5], Vaz et al. [6], Kalita et al. [7], Düğenci et al. [8].

The proved efficiency of sandwich beams and its current usage in a growing rate demands a higher level of acknowledgment of the mechanical properties, even when the structure is submitted to dynamic loads. For household refrigerators and food freezers, one of the main complaints to the customer care centers is related to noise generation, that is related most of the times with vibration of the cabinet that produces sound irradiation from internal components like shelves and containers, leaking to the outside of the unit. The efficient numerical models are necessary to estimate the dynamical behavior of such systems. When the complex sandwich beams are used the physical parameters are difficult to be estimated. Sandwich structures have high specific stiffness and strength. Because of these properties they are widely used in engineering and many types of sandwich structures has been studied with mathematical models. However, little attention has been paid to identifying the mechanical properties of these beams, [9]. Sandwich structures formed by polymeric thin sheet and lightweight core are widely used in engineering fields where there are required high bending stiffness and strength combined with a reduced weight. Nevertheless, the sandwich structures can be used in several engineering applications only if their elastic and damping properties are properly characterized. The heterogeneity e orthotropy this kind of structures, increase the difficulty to identify their constitutive parameters in comparison usual materials such as metals. In this case, an effective parameter identification method for sandwich structures is highly desirable.

A method using harmonic spatial field as primary input to identification of effective sandwich structural properties via an inverse wave approach was presented by Ichchou et al. [10]. The focus was on flexural vibration of symmetric honeycomb beam and panels. Another inverse parameter estimation method using an objective function taking acount the difference between numerical and experimental modal data was used by Matter et al. [11]. Damanpack et al. [12] presented a new finite element formulation for high-order impact analysis of sandwich beams with any boundary conditions subjected to simultaneous multiple small projectiles. The governing partial differential equations of motion are driven by Hamilton's principle. The elemental matrices are derived for a sandwich beam element that carrying four sprung masses on both face sheets. Alvelid [13] developed a 6 th order differential equation for the dynamic analysis of the deflection of a three-layer sandwich beam with a viscoelastic middle layer. In this study are considered the transverse shear deformation and rotational inertia effects of the covering layers. The boundary conditions applied in the covering layers are the same the Euler-Bernoulli case.

Damanpack and Khalili [14] investigated high-order free vibration of three-layered symmetric sandwich beam using dynamic stiffness method. The formulation used to connect axial and bending deformations in equations that representing the motion of an element is derived of Hemilton's principle. For the harmonic motion by considering the symmetrical sandwich beam, these equations were divided into two ordinary differential equations.Bekuit et al. [15] presented a quasi-two-dimensional finite element formulation for the static and dynamic analysis of sandwich beams. In this work, cubic Lagrange polynomial were used to interpolate the longitudinal and transverse displacement of each layer. The formulation was effective to evaluate the sandwich beam with viscoelastic core.

Various authors (Singh et al. [16], Pintelon et al. [17], Caracciolo et al. [18], Yang et al. [19], Park [20], Kim and Kreider [21], Chang [22], Bakström and Nilsson [23], Zhen and Wanji [24], Tagarielli et al. [25], Ivañez et al. [26]) have presented various experimental models and analysis to identify the physical parameters of a beam sandwich.

\section{THEORY}

\subsection{Optimization Methods}

\subsubsection{Genetic Algorithms (GA)}

The GA allows to reach the maximum of a function $f(x(i, j))$ subject to the following design constraints:

$x_{L}(i) \leq x_{i} \leq x_{U}(i) \Rightarrow i=1,2, \ldots, m$

where $x_{i}$ is the individuals (particles) set of design variables $x(i, j)$ which lower limit is $x_{L}(i)$ and the upper limit is $x_{U}(i)$ and $m$ is the number of design variables to be defined.

The GA represents the design variables as sets of binary numbers of $n b$ bits that are called chromosomes. This way, the viable interval for each variable $x_{i}$ is divided into $2^{n b}-1$ intervals. So, each variable $x_{i}$ can be represented by any discreet representation, for example, through a binary number: 11101 . This number can be decoded as:

$$
\begin{aligned}
& x_{i}=x_{L}(i)+\left(1 \cdot 2^{4}+1 \cdot 2^{3}+1 \cdot 2^{2}+0 \cdot 2^{1}+1 \cdot 2^{0}\right) \cdot S_{i} \text { or } \\
& x_{i}=x_{L}(i)+29 S_{i}
\end{aligned}
$$

where $S_{i}$ is the variable interval $x_{i}$ defined by

$$
S_{i}=\left(x_{U}(i)-x_{L}(i)\right) / 31
$$

This procedure defines the codification and decodification processes of the GA variables. The first step in the development of the GA is the creation of an initial population. Each individual $i$ of the population is a set with $m \times n b$ bits. The set of variables, $x_{i}$, in the binary form, creates the genetic code for each individual. An initial population with a $z$ size is created by a random process. The second step is to decode the genetic code of each individual using the Eqn. (2) and verifying the value correspondent to its respective fitness, i.e., of its objective functions, given by $F_{o b j l}, F_{o b j 2}, \ldots, F_{o b j z}$. The most fit individuals, with higher $F_{o b j}$ values are considered most "optimal". Their fitness value must reflect the design and the restrictions imposed on the problem being studied.

The third step includes the selection and crossover phases. Here, the weaker individuals are replaced by the stronger ones. The selection enables the best individuals to survive and serve as parents for the next project generation, through the fitness calculation. In this phase, the individuals are shuffled and have their order changed. After shuffling, two individuals are chosen. These are called parents and the one with the best fitness will originate from a child and will have some of its chromosomes randomly changed. This procedure is carried out $z$ times, generating the number of $z$ children. The crossover transference makes parts of the parents' chromosomes to create the next generation of the project by combining features in such a way as to create better 
individuals in average, but not always. The fourth step may switch a chromosome bit of an individual to its opposite value (e.g... 0 to 1 ). This step is called mutation and aims to introduce a new beneficial feature that does not exist in the current population. The mutation makes the fitness value of a child to suffer a change. This procedure is performed to avoid local optimum in search spaces induced by the random fitness change of the individual. The probability of a member bit to suffer mutation must vary between 0.005 and 0.1 , which demonstrates that this will rarely occur in the nature. If the feature introduced is not beneficial to the individual that suffered such mutation, probably it will not survive a future transformation step [2].

The fifth and last step verifies if the genetic code of the individual with the best fitness was replicated in any of the created children. If this does not happen, a child is randomly removed and the individual with the best fitness is added. This step improves the optimization accuracy. This operation is called elitist reproduction.

In order to conclude this generation, the highest $F_{o b j}$ value and its respective set of chromosomes $x_{i}$ are stored. If the required number of generations in this project is reached, the evolution will be concluded; otherwise, a new decodification step will begin, and continue successively. All the described process transforms an initial randomly chosen population into a population that is more adapted to its environment, making them more optimal.

\subsubsection{Differential Evolution (DE)}

The DE method comprises to create a vector for the initial population $x(i, j)_{g}$ with $j$ particles is randomly created and must be contained inside the solution space for the problem.

$x(i, j)_{g} \quad$ with $i=1,2, \ldots m ; j=1,2, \ldots z$ and $g=1,2, \ldots, \mathrm{G}$

(4)

where $i$ is the number of design variables and $g$ is the number of generations to be evaluated.

The DE creates a new parameters vector by adding the weighted difference between two particles of the population into a third. This operation is called mutation. The individual that comes from the mutation is mixed with the parameters from another predetermined particle called target vector, producing the trial vector. The parameters mixture is mentioned in the literature as crossover and will be explained in more details below. If the trial vector produces an upper value for the $F_{o b j}$ than the target vector, then the trial vector will replace the target vector in the following generation. This last operation is called selection. Each population vector has to serve a target vector once, so that $z$ competitions occur in a generation. The mutation and crossover strategies for the DE are described below.

A mutant vector $v(i, j)_{g+1}$ is created for each target vector $x(i, j)_{\mathrm{g}}$ randomly chosen within the population $z$ according to:

$v(i, j)_{g+1}=x_{t g 1},{ }_{g}+F\left(x_{t g 2},{ }_{g}-x_{t g 3}, g\right)$

In Eqn. (5), $x_{t g l, g}$ is the target vector. The indexes $\operatorname{tg} 1, \operatorname{tg} 2$ and $\operatorname{tg} 3 \in\{1,2, \ldots \mathrm{z}\}$ are integral and different among themselves. The integral ones $t g 1, \operatorname{tg} 2$ and $\operatorname{tg} 3$ randomly chosen are different from the index $j$ in such a way that $z$ must be larger or equal to 4 in order to allow for this condition. Factor $F$, called of mutant vector, controls the amplification of the differential variation $\left(x_{t g 2, g}-x_{t g 3, g}\right)$ is real and constant $\in[0,2]$, (see Ref. [27]).
In order to increase the diversity of the particles, the crossover is introduced. For this purpose, the trial vector is:

$u(i, j)_{g+1}=\left[u(1, j)_{g+1}, u(2, j)_{g+1}, \ldots, u(m, j)_{g+1}\right]$

where

$$
\begin{aligned}
& u(i, j)_{g+1}=v(i, j)_{g+1} \Rightarrow \operatorname{if}\left(r_{1}(i) \leq C r\right) \text { or } i=r_{2}(j) \text { or } \\
& u(i, j)_{g+1}=x(i, j)_{g} \Rightarrow \operatorname{if}\left(r_{1}(i)>C r\right) \text { and } i \neq r_{2}(j)
\end{aligned}
$$

In Eqn. (7ab) $r_{1}(i)$ is a real number chosen randomly $\in[0,1]$, $\mathrm{Cr}$ is a crossover rate $\in[0,1]$ and $r_{2}(i)$ is a randomly chosen index $\in[1,2, \ldots, m]$, which guarantees that $u(i, j)_{\mathrm{g}+1}$ receives at least a parameter of $v(i, j) g+1,[27]$.

The selection, the last step of the process, decides if the trial vector $u(i, j)_{\mathrm{g}+1}$ should or not become a member of the generation $g+1$. In this step $\mathrm{u}(i, j)_{\mathrm{g}+1}$ is compared to the target vector $x(i, j) g$ using the best fitness criterion, i.e.:

$$
x(i, j)_{g+1}=u(i, j)_{g+1} \Rightarrow \text { if } F_{o b j}\left(u(i, j)_{g+1}\right)>F_{o b j}\left(x(i, j)_{g}\right)
$$

or $\quad x(i, j)_{g+1}=x(i, j)_{g} \Rightarrow$ if $F_{o b j}\left(u(i, j)_{g+1}\right) \leq F_{o b j}\left(x(i, j)_{g}\right)$

\subsubsection{Particle Swarm Optimization (PSO)}

The first step for the implementation of the PSO consists of randomly generating a population with $\mathrm{m}$ particles and their corresponding initial speeds:

$$
\begin{aligned}
& x_{0}=x(i, j)_{0} \quad \text { with } \quad i=1,2, \ldots m \text { and } j=1,2, \ldots z \\
& v_{0}=v(i, j)_{0}
\end{aligned}
$$

where $m$ is the number of design variables and $j$ is the number of particles or population size.

The initial population, $x_{0}$, may be conveniently randomly chosen by employing the lower limit and the upper limit for each design variable the following way:

$$
x(i, j)_{0}=x_{L}(i)+r(i) \cdot\left(x_{U}(i)-x_{L}(i)\right) \quad \text { with } j=1,2, \ldots z
$$

where $x_{L}(i)$ denotes the lower limit for the design variable $i$, $x_{U}(i)$ denotes the upper limit and $r(i)$ indicates a randomly chosen number $\in[0,1]$ for each particle $j$.

The vector initial speed can be randomly created for each particle of this population according to the expression:

$$
v(i, j)_{0}=r(i) \cdot\left(x_{U}(i)-x_{L}(i)\right) / \gamma
$$

where $\gamma$ is a scale factor to be determined with the aim of limiting the size of the initial speed.

The original proposition of the PSO was also used in this work to compute the speeds of the particles at the instant $g+1, v(i, j)_{g}$. Its mathematical expression is:

$$
v(i, j)_{g+1}=v_{w}(i, j)_{g}+v_{i b}(i, j)_{g}+v_{k b}(i, j)_{g}
$$

where

$$
\begin{aligned}
& v_{w}(i, j)_{g}=w \cdot r(i) \cdot v(i, j)_{g} \\
& v_{i b}(i, j)_{g}=\alpha \cdot r(i) \cdot\left(x_{i b}(i, j)-x(i, j)_{k}\right) \\
& v_{k b}(i, j)_{g}=\beta \cdot r(i) \cdot\left(x_{g b}(i, j)-x(i, j)_{k}\right)
\end{aligned}
$$


$w$ is the inertia parameter, $x_{i b}(i, j)$ is the best location for the particular $i$ up to the last iteration $z$ (best particle), $x_{g b}(i, j)$ is the best result of all till the last iteration $z$ (global best), $\alpha$ and $\beta$ are two selected parameters $\in[0,1]$. The parcels that compose the speed at the instant $g+1$ are interpreted as follows [28]:

$v_{w}(i, j)_{g} \Rightarrow$ represents its tendency to continue moving along the same direction of the previous iteration;

$v_{i b}(i, j)_{g} \Rightarrow$ represents the tendency of the individual to return to its best location so far;

$v_{k b}(i, j)_{g} \Rightarrow$ represents the tendency of the particle to move towards the position of the best results of all the particles till the previous iteration.

Although directions are well defined in the PSO, the random character of their magnitudes has ensured search paths are able to avoid local maxima even when using relatively small populations [6]. New populations are created as the number of iterations increase. The particles that are converging towards the problem solution have their speeds converging towards the null value.

The new position $x(i, j)_{g+1}$ of each particle of the new population is calculated by employing the position $x(i, j)_{g}$ at the previous instant, and the speed $v(i, j)_{g+l}$. This new position is given by:

$$
x(i, j)_{g+1}=x(i, j)_{g}+v(i, j)_{g+1}
$$

For each new position of the particle is necessary to verify if the particle is located within the design region, i.e., if $x_{L}(i) \leq$ $x(i, j)_{g+1} \leq x_{U}(i)$. If the new particle is not within the design region, a new position for this particle is randomly created using Eq. (11).

\subsection{Two-dimensional elastic model}

The two-dimensional elastic analysis of sandwich beam was performed taking the assumptions that the materials are homogeneous with linear behavior and that in the interfaces the materials are perfectly bonded. The continuity of the displacements at the interfaces of the materials that make up the sandwich beam (continuous and fully glued interfaces) allows assemble the finite elements in the conventional way, even with different materials. In this work cubic Lagrangian Finite Element (16 nodes) for analyzes and the elastic components of the displacement $u=(u, v)$ can interpolated as follows:

$$
\begin{aligned}
& \left\{\begin{array}{c}
u(\mathrm{x}) \\
v(\mathrm{x})
\end{array}\right\} \cong\left[\begin{array}{ccccc}
N_{1}(\mathrm{x}) & 0 & \ldots & N_{16}(\mathrm{x}) & 0 \\
0 & N_{1}(\mathrm{x}) & \ldots & 0 & N_{16}(\mathrm{x})
\end{array}\right]\{\mathbf{q}\} \quad \text { or } \\
& \left\{\begin{array}{l}
u(\mathrm{x}) \\
v(\mathrm{x})
\end{array}\right\} \cong[\mathbf{N}(\mathbf{x})]\{\mathbf{q}\}
\end{aligned}
$$

where ' $\mathbf{x}$ ' is the coordinate along the beam axis $\{\mathbf{q}\}=\left\{u_{1}, v_{1}, \ldots, \quad u_{16}, \quad v_{16}\right\}^{\mathrm{T}}$ are the pairs $\left(u_{\mathrm{k}}, v_{\mathrm{k}}\right)$ that representing the displacement components at node $\mathrm{k}$ of the element and $N_{\mathrm{k}}(\mathrm{x})$ represent the Lagrange interpolation functions, well-known in the classical books of finite elements [29-30]. In this case, the Lagrange interpolations function for two dimensional cubic element with $n$ and $m$ nodes in $\xi$ and $\chi$ directions, respectively.

$\left[N_{k}\right]=L_{a}(\xi) L_{b}(\chi)$

$$
\begin{aligned}
& L_{a}(\xi)=\prod_{i=0,(i \neq a)}^{n}\left[\left(\xi-\xi_{i}\right) /\left(\xi_{a}-\xi_{i}\right)\right] \Rightarrow a=1,2, \ldots, \mathrm{k} \\
& L_{b}(\chi)=\prod_{j=0,(j \neq b)}^{m}\left[\left(\chi-\chi_{j}\right) /\left(\chi_{b}-\chi_{j}\right)\right] \Rightarrow b=1,2, \ldots, \mathrm{k}
\end{aligned}
$$

The linear strain vector is related to the generalized displacement vector in the following manner:

$$
\{\varepsilon\}=\left\{\begin{array}{c}
\varepsilon_{x x} \\
\varepsilon_{y y} \\
\gamma_{x y}
\end{array}\right\}=\left\{\begin{array}{c}
\partial u(\mathbf{x}) / \partial x \\
\partial v(\mathbf{x}) / \partial y \\
\partial u(\mathbf{x}) / \partial y+\partial v(\mathbf{x}) / \partial x
\end{array}\right\} \cong[\mathbf{B}]\{\mathbf{q}\}
$$

where $[\mathbf{B}]$ is a $(3 \times 32)$ matrix relating the three strains to the generalized displacements and is given by:

$$
[\mathbf{B}]=\left[\begin{array}{ccccc}
\partial N_{1} / \partial x & 0 & \ldots & \partial N_{16} / \partial x & 0 \\
0 & \partial N_{1} / \partial y & \ldots & 0 & \partial N_{16} / \partial y \\
\partial N_{1} / \partial y & \partial N_{1} / \partial x & \ldots & \partial N_{16} / \partial y & \partial N_{16} / \partial x
\end{array}\right]
$$

The element stiffness matrix, $[\mathbf{K}]$, is obtained by integration and its expression is provided by Eq. (24), see Refs. [29-30].

$$
[\mathbf{K}]=\int_{A}[\mathbf{B}]^{\mathrm{T}}[\mathbf{D}][\mathbf{B}] t_{e} d A
$$

where $t_{e}$ denotes the thickness of the element, $A$ denotes the area of the element and [D] is the $(3 \times 3)$ matrix called constitutive matrix, which relates three stresses and three strains,

$\{\sigma\}=\left\{\begin{array}{lll}\sigma_{x x} & \sigma_{y y} & \tau_{x y}\end{array}\right\}^{\mathrm{T}}=[\mathbf{D}]\left\{\begin{array}{lll}\varepsilon_{x x} & \varepsilon_{y y} & \gamma_{x y}\end{array}\right\}^{\mathrm{T}}=[\mathbf{D}]\{\varepsilon\}$

where

$$
[\mathbf{D}]=\frac{E}{1-v^{2}}\left[\begin{array}{ccc}
1 & v & 0 \\
v & 1 & 0 \\
0 & 0 & (1-v) / 2
\end{array}\right],
$$

In Eqn. (26) E is the modulus of elasticity and $v$ is Poisson's ratio. The $[\mathbf{D}]$ matrix is written as a function of the elastic material parameters and the adjustment of these parameters as a function of angular frequency $(\omega)$ was the main objective of this work. The mass matrix is obtained using the following integral:

$$
[\mathbf{M}]=\int_{A} \rho[\mathbf{N}]^{\mathrm{T}}[\mathbf{N}] t_{e} d A
$$

where $\rho$ is the material density and $[\mathbf{N}]$ is a matrix with order $(2 \times 32)$ of Eq. $(18)$.

For punctual external forces as $\mathrm{F}(\mathrm{x}, \mathrm{t})=\mathrm{F} 0 \sin (\omega \mathrm{t})$ acting on node $\mathrm{k}$ and the balance of the element to the harmonic analysis can be written in the following form [29-30]:

$\left[\mathbf{K}_{e}(E, \omega)\right]\{\mathbf{q}\}=\{\mathbf{F}\}$

where

$$
\left[\mathbf{K}_{e}(\mathrm{E}, \omega)\right]=[\mathbf{K}(\mathrm{E})]-\omega^{2}[\mathbf{M}],
$$

$\{\mathbf{F}\}=\left\{\begin{array}{lll}0 \ldots & F_{0} & \ldots 0\end{array}\right\}^{\mathrm{T}}$

where 
and the position is not equal to zero at $2 \mathrm{k}$ when the force is applied in the ' $\mathrm{y}$ ' direction and $2(\mathrm{k}-1)$ when the force is applied in the ' $x$ ' direction.

The detail of how the boundary condition (clamped) was imposed in the end of sandwich beam is showed in Figure 1. As can be seen, this condition is much better represented with two-dimensional elements, because null displacements are imposed only on the nodes in contact with the rigid base. The Figure 2 shows the pattern construction of the homogeneous finite element mesh (shown only a band of 3 $\mathrm{mm}$ thickness along the height of the beam). Note that elements with a length of $3 \mathrm{~mm}$ were used to keep the aspect ratio limited to 5 for the elements of the steel sheet.

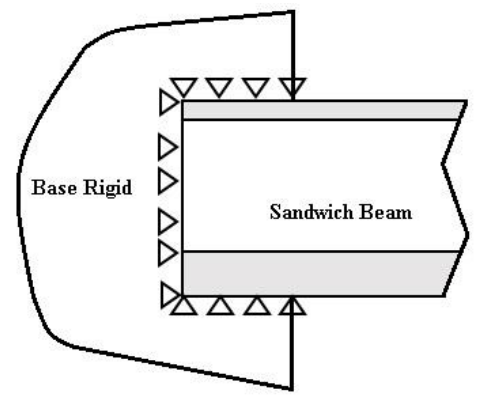

Fig. 1: Boundary conditions for the clamped end.
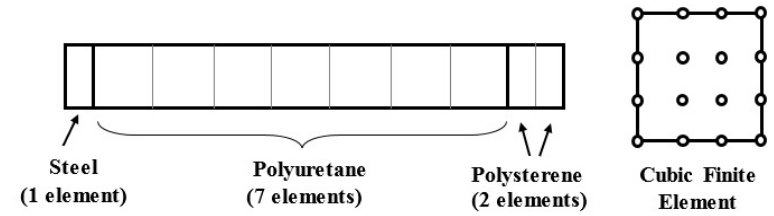

Fig. 2:Construction pattern mesh along the thickness of the beam (rotated $90^{\circ}$ ) and cubic Lagrangian element used in the analysis.

\section{RESULTS}

The main objective of this work is evaluating the quality of the solutions obtained with the parametric optimization to estimate the core shear modulus $\left(G_{p}\right)$ and the core loss factor $\left(\eta_{p}\right)$ of the PRF and the elasticity modulus $\left(E_{h}\right)$ and the loss factor $\left(\eta_{h}\right)$ of the HIP. The physical parameters are correlated by the complex elasticity modulus by:

$$
E^{*}=E(1+\mathrm{j} \eta)
$$

According Backström and Nilsson [23], the PRF does not has significant influence on the dynamic behavior of sandwiches beams, it is assumed that the classical relationship between $E$ and $G$ given by Eq. (32) is valid.

$$
E=2(1+v) G
$$

where $v$ is the Poisson coefficient and its value is 0,3 .

The optimization methods applied in this work are: GA, DE and PSO. The strategy of evaluation these properties will be conducted in 2 steps: a) Step I - Experimental evaluation of the FRF and b) Step II - Optimization of the physical parameters. The present methodology is applied two sandwich beams, one without and another with superficial modification. The experimental sandwich beam structure used in this work is made with the association of hot-rolled steel, PRF and HIP with thickness of $0.6 \mathrm{~mm}, 38.25 \mathrm{~mm}$ and
$1.25 \mathrm{~mm}$, respectively, (see Fig. 3). The beam width is 39.18 $\mathrm{mm}$.

\subsection{Step I-Experimental evaluation of FRF}

The first step of this methodology is to evaluate experimentally the FRF of the sandwich beam denoted by FRFexp. This is done with the evaluation of FRF between an impulsive signal and its vibrational response. The experimental data are obtained using the impact hammer and four accelerometers displaced along the sample $\left(A_{1}, A_{2}, A_{3}\right.$ and $\mathrm{A}_{4}$ ), Fig. 3. The impact force was applied in the position of the accelerometer $\mathrm{A}_{2}$ on the steel side and Fig. 4 shows the FRF curve obtained. Table 1 shows the values of these parameter to the accelerometer $\mathrm{A}_{2}$ and the natural frequencies obtained using $2 \mathrm{D}$ elasticity theory.

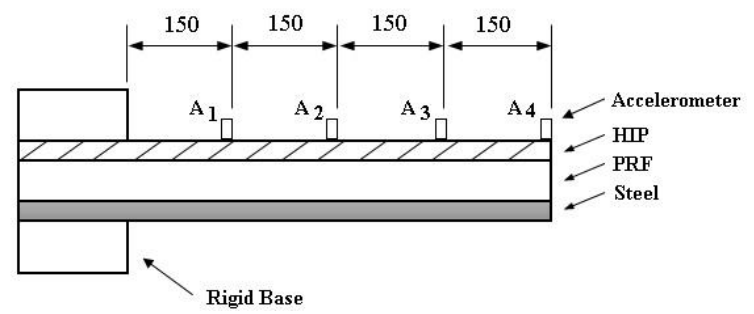

Fig. 3:Beam Structure (Dimensions in $\mathrm{mm}$ ).

\subsection{Step II - Optimization of the $F_{o b j}$}

The mathematical model is obtained using $2 \mathrm{D}$ elasticity theory and the physical parameters $\left(G_{\mathrm{p}}, E_{h}, \eta_{p}\right.$ and $\left.\eta_{h}\right)$, are estimated through the adjustment of the numerical FRF $\left(F R F_{\text {num }}\right)$ generated by computer simulation of the finite element model coupled to methods GA, PSO and DE. The parameters were optimized considering three different frequency ranges around the natural frequencies $\left(f_{n}\right)$ of the the first three modes of vibration. The parameters used in this application by three methods of optimization are showed in Table 2 . The finite element mesh is composed by 775 nodes and 80 elements. The objective function $F_{o b j}$ is evaluated in frequency range defined between $\mathrm{f}_{1}$ and $\mathrm{f}_{2}$, denote by

$F_{o b j}=\sum_{\mathrm{f}_{1}}^{\mathrm{f}_{2}}\left|F R F_{\text {num }}-F R F_{\text {exp }}\right|$

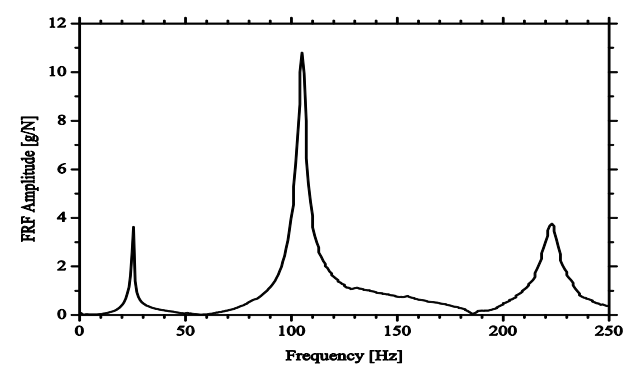

Fig. 4:FRF curve of accelerometer $A_{2}$.

Table 1. Experimental and numeric natural frequencies

\begin{tabular}{|c|c|c|c|}
\hline \multirow{2}{*}{$\begin{array}{c}\text { Mode } \\
\text { shape }\end{array}$} & \multicolumn{3}{|c|}{ Natural Frequencies $\left(\mathrm{f}_{\mathrm{n}}\right)$ in $[\mathrm{Hz}]$} \\
\cline { 2 - 4 } & Experimental $\mathrm{A}_{2}$ & 2D elasticity & Error (\%) \\
\hline 1 & 24.76 & 24.50 & -1.06 \\
\hline 2 & 104.34 & 107.36 & +2.81 \\
\hline 3 & 221.87 & 228.50 & +2.90 \\
\hline
\end{tabular}


Table 2. GA, DE and PSO parameters.

\begin{tabular}{|c|c|c|c|}
\hline Parameter & GA & DE & PSO \\
\hline Population size & 20 & 20 & 20 \\
\hline $\begin{array}{c}\text { Number of evaluation of the } \\
F_{o b j}\end{array}$ & 3000 & 3000 & 3000 \\
\hline Crossover rate & 0.9 & 0.85 & - \\
\hline Mutation rate & 0.02 & - & - \\
\hline Number of Cromossomes & 12 & - & - \\
\hline Aplification Factor $(F)$ & - & 0.5 & - \\
\hline Inertia weight $(w)$ & - & - & 0.01 \\
\hline$\alpha / \beta / \gamma$ & - & - & $0.9 / 0.6 / 0.5$ \\
\hline
\end{tabular}

Pritz [31] in the study of damping behavior of the solid material used for sound and vibration control verified that $E$, $G$ and $\eta$ are frequency dependents and $\eta$ is more susceptible to frequency variation. Fact also observed by Barbieri et al. [32] when evaluated statically and dynamically the parameters of one specimen made of the HIP and another the PRF in three frequency bands. Furthermore, the authors evaluated dynamically a sandwich beam excited by a mini shaker and the physical parameters of the HIP and PRF were estimated using the Timoshenko beam theory and two optimization methods: amplitude correlation coefficient (ACC) and GA, Table 3. Based on these studied, the lower and upper limit of the parameters $E$ and $G$ are determined as $\pm 30 \%$ of the GA results and the lower and upper limit of the parameter $\eta$ are $\pm 70 \%$ of the results.

The design constraints applied in the optimizations are shown in Table 4 . The physical parameters are adjusted for the first three vibration modes separately. The frequency range in parameter adjustment is chosen as being approximately $\pm 10 \mathrm{~Hz}$ of the $f_{n}$ values obtained in Step 1, Table 5. The frequency increment used in all adjustments is $0.25 \mathrm{~Hz}$. Figures 5-7 show the curves of the adjusted FRF $\left(F R F_{\text {num }}\right)$ and a comparison with the experimental FRF $\left(F R F_{\text {exp }}\right)$ for three first vibration modes. Figures 8-10 show the converge behavior of the function objective for each analysis.

Table 3. Parameter obtained by Barbieri et al. [32].

\begin{tabular}{|c|c|c|c|c|c|}
\hline \multirow{2}{*}{ Method } & \multicolumn{2}{|c|}{ HIP } & \multicolumn{3}{c|}{ PRF } \\
\cline { 2 - 6 } & $E_{h}[\mathrm{GPa}]$ & $\eta_{h}$ & $G_{p}[\mathrm{MPa}]$ & $E_{p}[\mathrm{MPa}]$ & $\eta_{p}$ \\
\hline GA & 1.583 & 0.045 & 3.287 & 3.292 & 0.064 \\
\hline ACC & 1.580 & 0.047 & 8.55 & 8.56 & 0.063 \\
\hline
\end{tabular}

Table 4. Design constraints.

\begin{tabular}{|c|c|c|c|}
\hline \multirow{2}{*}{ Material } & $\begin{array}{c}\text { Physical } \\
\text { Parameter }\end{array}$ & $\begin{array}{c}\text { Lower Limit } \\
x_{L}(i)\end{array}$ & $\begin{array}{c}\text { Upper Limit } \\
x_{U}(i)\end{array}$ \\
\hline \multirow{2}{*}{ HIP } & $E_{h}[\mathrm{Gpa}]$ & 1.11 & 1.79 \\
\cline { 2 - 4 } & $\eta_{h}$ & 0.014 & 0.076 \\
\hline \multirow{2}{*}{ PRF } & $E_{p}[\mathrm{Mpa}]$ & 5.98 & 11.11 \\
\cline { 2 - 4 } & $\eta_{p}$ & 0.019 & 0.110 \\
\hline
\end{tabular}

In all cases, the PSO method presented faster convergence than the other methods. The PSO method has been more efficient for this type of problem. However, all methods converged on a similar value of the objective function. There are not many works in the literature of parameters identification applied for sandwich beams which compare the efficiency of these three methods.

Table 5. Frequency range in $[\mathrm{Hz}]$.

\begin{tabular}{|c|c|c|c|}
\hline Mode shape & $\mathrm{f}_{1}$ & $\mathrm{f}_{\mathrm{n}}$ & $\mathrm{f}_{2}$ \\
\hline $1^{\text {st }}$ & 14.0 & 24.76 & 34.0 \\
\hline $2^{\text {nd }}$ & 94.0 & 104.34 & 114.0 \\
\hline $3^{\text {rd }}$ & 212.0 & 221.87 & 332.0 \\
\hline
\end{tabular}

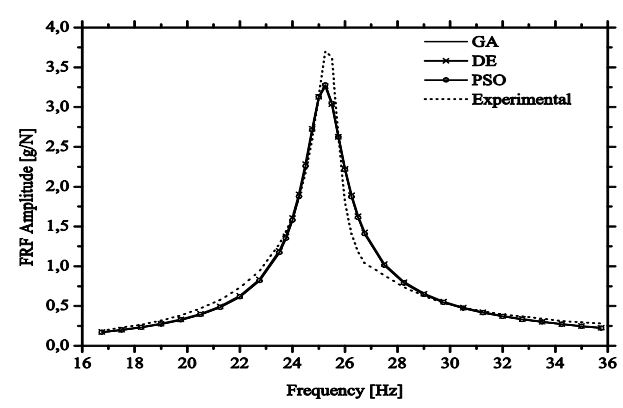

Fig. 5: $F R F_{n u m}$ for first mode.

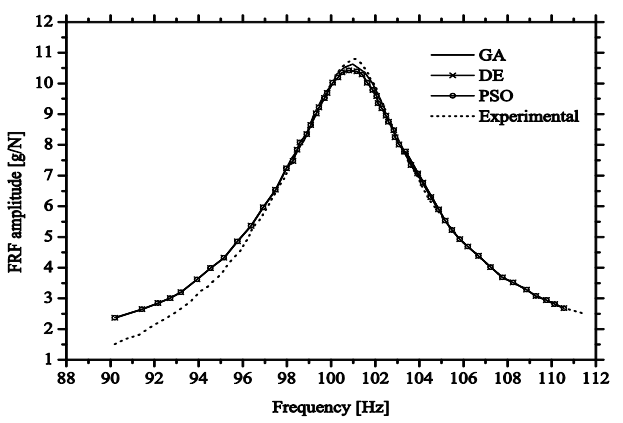

Fig. 6: $F R F_{n u m}$ for second mode.

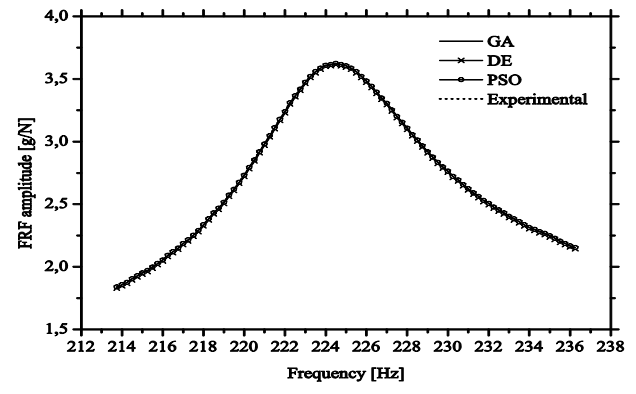

Fig. 7:FRF $F_{\text {num }}$ for third mode.

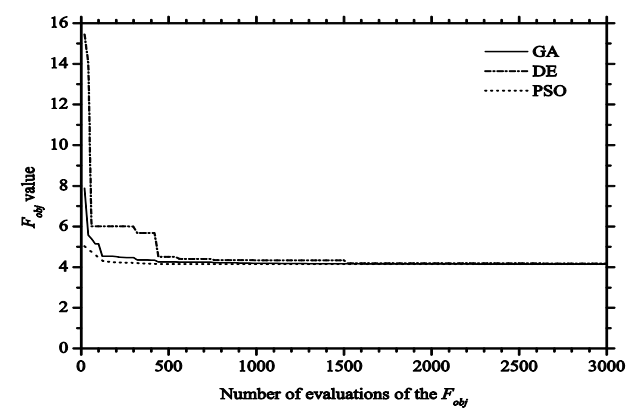


Fig. 8: Convergence behavior for first mode.

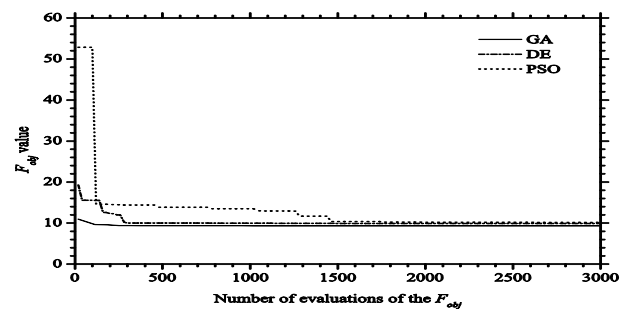

Fig. 9:Convergence behavior for second mode.

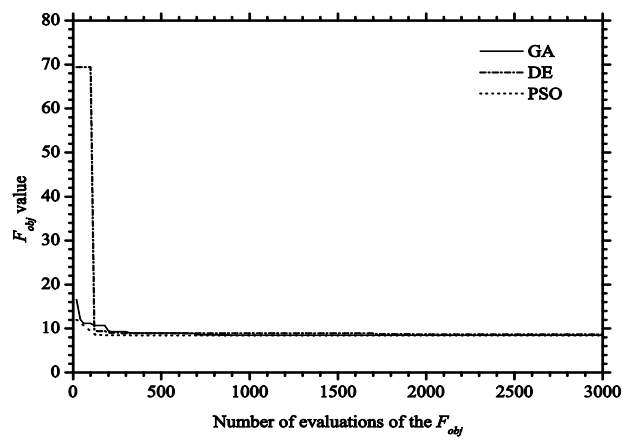

Fig. 10: Convergence behavior for third mode.

Table 6 shows the values of the optimal physical parameters for the HIP and PRF. It can be noted that the loss factor $(\eta)$ of the two materials presented large differences for adjustment around the regions of the second and third vibration modes. The largest variations of Young's modulus of the PRF values were obtained for the region of the third vibration mode.

Table 6. Optimal Results.

\begin{tabular}{|c|c|c|c|c|c|c|c|c|c|}
\hline \multirow{3}{*}{$\begin{array}{l}\frac{0}{0} \\
\frac{\pi}{5} \\
\frac{0}{2} \\
\frac{0}{\Sigma}\end{array}$} & \multirow{3}{*}{ 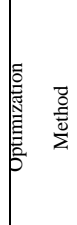 } & \multicolumn{4}{|c|}{$\begin{array}{l}\text { High Impact Polystyrene } \\
\text { (HIP) }\end{array}$} & \multicolumn{4}{|c|}{$\begin{array}{l}\text { Polyurethane rigid foam } \\
\qquad \text { (PRF) }\end{array}$} \\
\hline & & \multicolumn{2}{|c|}{$\begin{array}{l}\text { Timoshenko } \\
\text { beam theory* }\end{array}$} & \multicolumn{2}{|c|}{ 2D elasticity } & \multicolumn{2}{|c|}{$\begin{array}{l}\text { Timoshenko } \\
\text { beam theory* }\end{array}$} & \multicolumn{2}{|c|}{ 2D elasticity } \\
\hline & & $\begin{array}{c}E_{h} \\
\mathrm{GPa}\end{array}$ & $\eta_{h}$ & $\begin{array}{c}E_{h} \\
\mathrm{GPa}\end{array}$ & $\eta_{h}$ & $\begin{array}{l}E_{p} \\
\text { MPa }\end{array}$ & $\eta_{p}$ & $\begin{array}{r}E_{p} \\
\mathrm{MPa}\end{array}$ & $\eta_{p}$ \\
\hline \multirow{3}{*}{$1^{\text {st }}$} & GA & & & 1.28 & 0.042 & \multirow{3}{*}{9.47} & \multirow{3}{*}{0.064} & 8.79 & 0.063 \\
\hline & PSO & 1.40 & 0.044 & 1.28 & 0.046 & & & 8.71 & 0.051 \\
\hline & DE & & & 1.30 & 0.048 & & & 8.26 & 0.048 \\
\hline \multirow{3}{*}{$2^{\text {nd }}$} & GA & \multirow{3}{*}{1.40} & \multirow{3}{*}{0.044} & 1.42 & 0.023 & \multirow{3}{*}{9.47} & \multirow{3}{*}{0.064} & 9.49 & 0.044 \\
\hline & PSO & & & 1.40 & 0.023 & & & 9.63 & 0.043 \\
\hline & $\mathrm{DE}$ & & & 1.43 & 0.020 & & & 9.43 & 0.047 \\
\hline \multirow{3}{*}{$3^{\text {rd }}$} & GA & \multirow{3}{*}{1.40} & \multirow{3}{*}{0.044} & 1.38 & 0.016 & \multirow{3}{*}{9.47} & \multirow{3}{*}{0.064} & 7.03 & 0.025 \\
\hline & PSO & & & 1.38 & 0.016 & & & 7.03 & 0.025 \\
\hline & $\mathrm{DE}$ & & & 1.39 & 0.016 & & & 7.01 & 0.025 \\
\hline
\end{tabular}

Figures 11 to 13 shows the three mode shapes (displacement in the $\mathrm{x}$ direction) of the system using the numeric 2D elasticity model. It is possible to notice, mainly in the Figs. 11 and 13, regions where the thickness are extended and compressed. This type of behavior is not possible to be obtained with the Thimoshenko beam model.
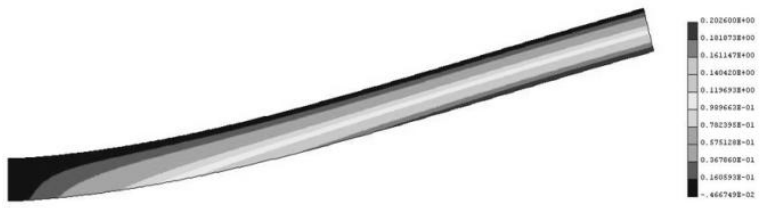

Fig. 11:First mode shape.

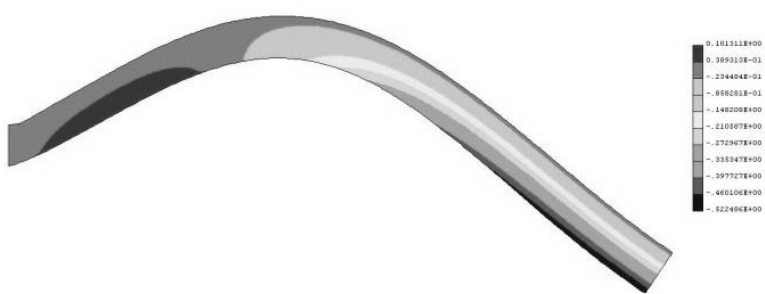

Fig. 12:Second mode shape.

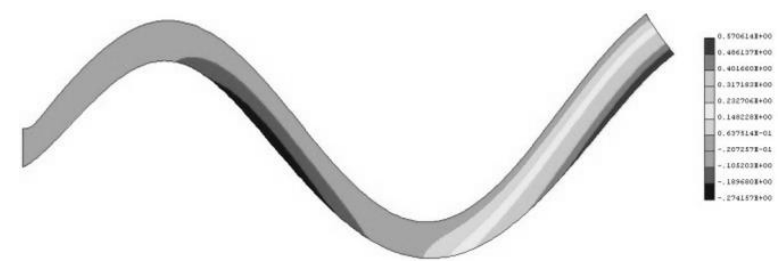

Fig. 13: Third mode shape.

\subsection{Sandwich beam with local modification}

Finally, the methodology is used to optimize the parameters of a sandwich beam with local modification. The modification (perturbation) was introduced into the system by removing $2 \mathrm{~mm}$ from the face of polystyrene at the position of $75 \mathrm{~mm}$ from the rigid base. The finite element mesh is refined at modification region and it formed by 27,931 nodes and 3,000 elements. Figure 14 shows the experimental FRF curves of the system without and with modification. Notes a great variation in the first natural frequency and little variation in the third natural frequency. The second natural frequency practically was not changed.

Table 7 shows the value of the three first natural frequencies to the system without and with modification. In this example, the parameters are adjusted only for the first natural frequency with PSO. The results obtained after 3,000 evaluations of the $F_{o b j}$ are showed in Table 8. Figure 15 shows the curves of the adjusted FRF $\left(F R F_{\text {num }}\right)$ and a comparison with the experimental FRF $\left(F R F_{\text {exp }}\right)$ for the first vibration mode. Figure 16 shows the converge behavior of the function objective for PSO optimization. This example was intentionally included to show that the present methodology is efficient to evaluate the physical properties of the solid structures by parameter optimization.

Table 7. Experimental frequencies with and without modification.

\begin{tabular}{|c|c|c|}
\hline \multirow{2}{*}{ Mode shape } & \multicolumn{2}{|c|}{ Experimental $\mathrm{f}_{n}[\mathrm{~Hz}]$} \\
\cline { 2 - 3 } & Without & With \\
\hline $1^{\text {st }}$ & 24.76 & 13.40 \\
\hline $2^{\text {nd }}$ & 104.34 & 104.96 \\
\hline $3^{\text {rd }}$ & 221.87 & 219.50 \\
\hline
\end{tabular}


Table 8. Optimal results for sandwich beam without and with modification.

\begin{tabular}{|c|c|c|c|c|c|c|c|c|c|}
\hline \multirow{3}{*}{ 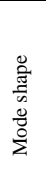 } & \multirow{3}{*}{ 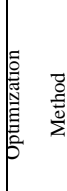 } & \multicolumn{4}{|c|}{$\begin{array}{c}\text { High Impact Polystyrene } \\
\text { (HIP) }\end{array}$} & \multicolumn{4}{|c|}{$\begin{array}{l}\text { Polyurethane rigid foam } \\
\text { (PRF) }\end{array}$} \\
\hline & & \multicolumn{2}{|c|}{ Without } & \multicolumn{2}{|c|}{ With } & \multicolumn{2}{|c|}{ Without } & \multicolumn{2}{|c|}{ With } \\
\hline & & $\begin{array}{c}E_{h} \\
\mathrm{GPa}\end{array}$ & $\eta_{h}$ & $\begin{array}{c}E_{h} \\
\mathrm{GPa}\end{array}$ & $\eta_{h}$ & $\begin{array}{c}E_{p} \\
\mathrm{MPa}\end{array}$ & $\eta_{p}$ & $\begin{array}{c}E_{p} \\
\mathrm{MPa}\end{array}$ & $\eta_{p}$ \\
\hline $1^{\mathrm{st}}$ & PSO & 1.28 & 0.046 & 1.45 & 0.040 & 8.71 & 0.051 & 8.89 & 0.058 \\
\hline
\end{tabular}

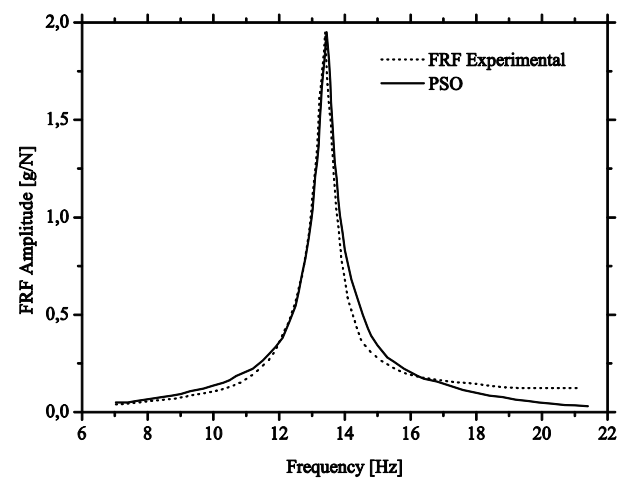

Fig. 15:FRF $F_{\text {num }}$ and $F R F_{\text {exp }}$ for first mode.

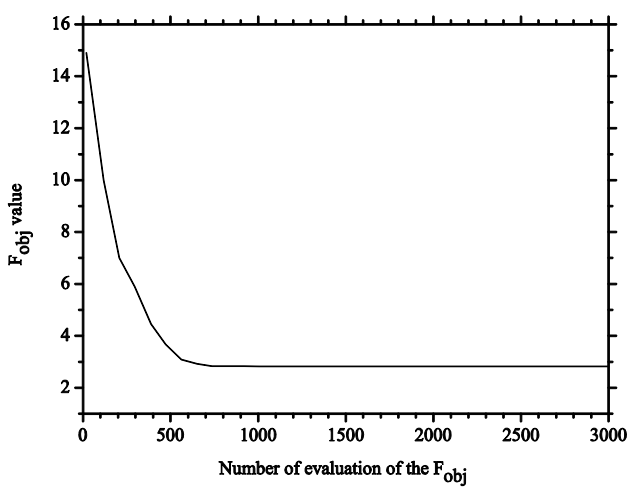

Fig. 16: Convergence behavior.

\section{CONCLUSIONS}

Based on the results it is possible to ensure that the mathematical model obtained with 2D elasticity is a good tool for representing the dynamic behavior of sandwich beams with and without local modifications. The 2D elasticity theory was used to update the values of physical parameters of mathematical models of sandwich beam made with the association of Hot-Rolled Steel, Polyurethane Rigid Foam and High Impact Polystyrene. The physical parameters estimated were the Young's modulus and loss factor of the of the Polyurethane Rigid Foam and the High Impact Polystyrene and was verified that the parameters are frequency dependent.

All optimization methods (Genetic Algorithm, Differential Evolution and Particle Swarm Optimization) presented good agreement when compared the optimized and experimental FRF curves. The Particle Swarm Optimization have shown faster convergence than others methods for this application.

\section{ACKNOWLEDGMENTS}

This work had financial support provided by FINEP (Case No 0-1-11-0132-00).

\section{REFERENCES}

[1] Holland J., 1975. Adaptation in Natural and Artificial System. University of Michigan Press, Ann Arbor.

[2] Goldberg D. E., 1989. Genetic Algorithms in Search, Optimization, and Machine Learning. Addison\&Willey, New York

[3] Storn R., Price K.,1995. Differential Evolution: a simple and efficient adaptive scheme for global optimization over continuous spaces. Int. Comp. Sci. Institute. Technical Report TR-95-012, Berkeley.

[4] Eberhart R. C., Kennedy J., 1995. A new optimizer using particle swarm theory. Proceedings of the $6^{\text {th }}$ Int. Symp. Micro Mach. Human Sci. IEEE Press, 39-43.

[5] Quaranta G., Monti G., Marano G. C., 2010. Parameters identification of Van der Pol-Duffing oscillators via particle swarm optimization and differential evolution. Mech. Syst. Signal Process, 24 (7), 2076-2095.

[6] Vaz Junior M., Cardoso E. L., Stahlschmidt J., 2013. Particle swarm optimization and identification of inelastic material parameters. Eng. Comput., 30 (7), 936-960.

[7] Kalita K., R. Chaudhari, and M. Ramachandran, "Mechanical Characterization and Finite Element Investigation on Properties of PLA-Jute Composite", International Journal of Computer Applications, 123 (2013): pp. 17-21.

[8] Düğenci M., Aydemir A., Esen I., Aydın M. E., 2015. Creep modelling of polypropylenes using artificial neural networks trained with Bee algorithms. Eng. Appl. Artif. Intell., 45, 71-79.

[9] Shi Y., Sol H., Hua H., 2006. Material parameter identification of sandwich beams by an inverse method. J. Sound Vib., 290, 1234-1255.

[10] Ichchou M. N., Bareille O., Berthaut J., 2008., Identification of effective sandwich structural properties via an inverse wave approach. Eng. Struct., 30 (10), 2591-2604.

[11] Matter M., Gmür T., Cugnoni J., Schorderet A., 2011. Identification of the elastic and damping properties in sandwich structures with a low core-to-skin stiffness ratio. Composite Struct., 93, 331-341.

[12] Damanpack A. R., Shakeri M., Aghdam M. M., 2013. A new finite element model for low-velocity impact analysis of sandwich beams subjected to multiple projectiles. Composite Struct., 104, 21-33.

[13] Alvelid M., 2013. Sixth order differential equation for sandwich beam deflection including transverse shear. Composite Struct., 102, 29-37.

[14] Damanpack A. R., Khalili S. M. R., 2012. High-order free vibration analysis of sandwich beams with a flexible core using dynamic stiffness method. Composite Struct., 94 (5), 1503-1514.

[15] Bekuit J. J. R. B., Oguamanam D. C. D., Damisa O., 2007. A quasi-2D finite element formulation for the analysis of sandwich beams. Finite Elem. Anal. Design, 43 (14), 1099-1107. 
[16] Singh R., Davies P., Bajaj A. K., 2003. Estimation of the dynamical properties of polyurethane foam through use of Prony series. J. Sound Vib., 264, 1005-1043.

[17] Pintelon R., Guillaume P., Vanlanduit S., Belder K., Rolain Y., 2004. Identification of Young's modulus from broadband modal analysis experiments. Mech. Syst. Signal Process, 18 (4), 699-726.

[18] Caracciolo R., Gasparetto A., Giovagnoni M., 2004. An experimental technique for complete dynamic characterization of a viscoelastic material. J. Sound Vib., 272, 1013-1032.

[19] Yang W. P., Chen L. W., Wang C. C., 2005. Vibration and dynamic stability of a traveling sandwich beam. J. Sound Vib., 285, 597-614.

[20] Park J., 2005. Transfer function methods to measure dynamic mechanical properties of complex structures. J. Sound Vib., 288, 57-79.

[21] Kim S., Kreider K. L., 2006. Parameter identification for nonlinear elastic and viscoelastic plates. Appl. Num. Math., 56 (12), 1538-1544.

[22] Chang W. D., 2006. An improved real-coded genetic algorithm for parameters estimation of nonlinear systems. Mech. Syst. Signal Proc., 20, 236-246.

[23] Backström D., Nilsson A. C., 2007. Modelling the vibration of sandwich beams using frequency-dependent parameters. J. Sound Vib., 300, 589-611.

[24] Zhen W., Wanji C., 2008. An assessment of several displacement-based theories for the vibration and stability analysis of laminated composite and sandwich beams. Composite Struct., 84, 337-349.

[25] Tagarielli V. L., Deshpande V. S., Fleck N. A., 2010. Prediction of the dynamic response of composite sandwich beams under shock loading. Int. J. Impact Eng., 37 (7), 854-864.

[26] Ivañez I., Santiuste C., Saez S. S., 2010. FEM analysis of dynamic flexural behaviour of composite sandwich beams with foam core. Composite Struct., 92, 22852291.

[27] Storn R., Price K., 1997. Differential evolution: a simple and efficient heuristic for global optimization over continuous spaces., J. Glob. Opt., 11 (4), 341-359.

[28] Blum C., Li X., 2008. Swarm intelligence in optimization: Introduction and Applications. Springer, Heildelberg.

[29] Zienkiewicz O. C., Taylor R. L., 2000. Finite Element Method: Volume 1 The Basis. Butterworth-Heinemann, Oxford.

[30] Logan D. L., 2011. A First Course in the Finite Element Method. Cengage Learning, Platteville.

[31] Pitz T., 2004. Frequency power law of material damping. Appl. Acoust, 65, 1027-1036.

[32] Barbieri N., Barbieri R., Winikes L. C., Oresten L. F., 2008. Estimation of parameters of a three-layered sandwich beam. J. Mech. Mater. Struct., 3 (3), 527-544. 